\title{
Pregnant patient presenting with syncope and a medulloblastoma: a case report
}

\author{
Mark Ishak, B.S. \\ New York College of Osteopathic Medicine of NYIT, Old Westbury, NY, USA \\ *Corresponding Author: Mark Ishak, mishak@nyit.edu
}

\begin{abstract}
Medulloblastoma is a primary cerebellar tumor seen most commonly in the pediatric population. In adults, it represents about $1 \%$ of cancer found in the central nervous system. In adult medulloblastoma, only one third of cases occur in women and even less in pregnant women. This case describes a 34-year-old pregnant woman who presented to the emergency department with the syncope, which was found to be secondary to a medulloblastoma.
\end{abstract}

Keywords: Adult medulloblastoma; pregnant; syncope.

\section{INTRODUCTION}

D espite the low incidence of cases of adult medulloblastoma, less than $33 \%$ of adult cases occur in women. On a hematoxylin and eosin (H\&E) stain, medulluloblastoma appears as a small blue cell tumor. It usually arises in the midline of the cerebellum in children, but tends to be lateral in adults. Symptoms include nausea, vomiting, loss of coordination, morning headache, seizures, personality changes, and unexplained weight loss or weight gain. Patients who undergo radiation therapy with adjuvant chemotherapy have a 5-year survival rate of about $58-84 \%{ }^{4}$

Medulloblastoma is a cerebellar granule cell tumor, the most common type of cell in the cerebellum, and its robust proliferative capacity is thought to be regulated by the Sonic Hedgehog (SHH) pathway., ${ }^{5,6}$ However, data suggests that cerebellar granule cells may be responsive to estrogen receptor stimulation. As such, pharmacologic blockade of estrogen receptors in cell cultures and xenograph models of medulloblastoma have been shown to inhibit the migration and growth of medulloblastoma. ${ }^{7}$ These data suggest that the high levels of estrogen observed during pregnancy may be a contributing factor for this tumor in pregnancy. ${ }^{7}$

\section{CASE PRESENTATION}

The patient is a previously healthy 34-year-old woman G4 P3013 who presented to the Brooklyn Hospital Center Emergency Department with the chief concern of head trauma following a fall 3 days prior to presentation. Along with her syncopal episode she had dizziness, mild head trauma, and approximately 2 minutes of loss of consciousness. Since the time of the syncopal episode and the fall, she experienced posterior headaches with worsening nausea and vomiting. She denied any fever, chills, abdominal pain, numbness and tingling, and vaginal bleeding. All labs were unremark- able. The patient was then admitted and an MRI and CT scan were ordered (Figures 1-4). The radiologist's impression of the imaging studies included a differential diagnosis of astrocytoma, due to the age of the patient and the associated symptoms. Also noted was a downward tonsillar herniation into the foramen magnum, which suggested an aggressive primary neoplasm. The result of CT scans also suggested astrocytoma based on its location, since it was compressing the fourth ventricle anteriorly and laterally. Also, the radiographic scans revealed some hydrocephalus and edema consistent with obstructive hydrocephalus from the herniation of the tonsils into the foramen magnum. T2 MRI also confirmed a mass in the cerebellum (Figures 3,4). This patient was then sent to surgery for resection of the mass in the cerebellum.

At the time of surgery, the surgeon's gross impression of the mass was meningioma, since the tumor did not have the typical consistency of medulloblastoma and was firm and circumscribed. Pathology was consulted for frozen section where the diagnosis was initially deferred (Figures 5 and 6). After histological evaluation and review, the slides of the lesion were sent for neuropathology consultation at Memorial Sloan-Kettering Cancer Center where a diagnosis of medulloblastoma consistent with desmoplastic type was rendered.

Postsurgically, the patient experienced complications with persistent hydrocephalus and intense headaches that were treated with the insertion of a ventriculoperitoneal shunt. The patient decided to terminate the pregnancy in her second trimester. Upon receiving her diagnosis and aborting the fetus, the patient received an oncology consult recommending radiation therapy; however, she discharged herself from the hospital against medical advice. 


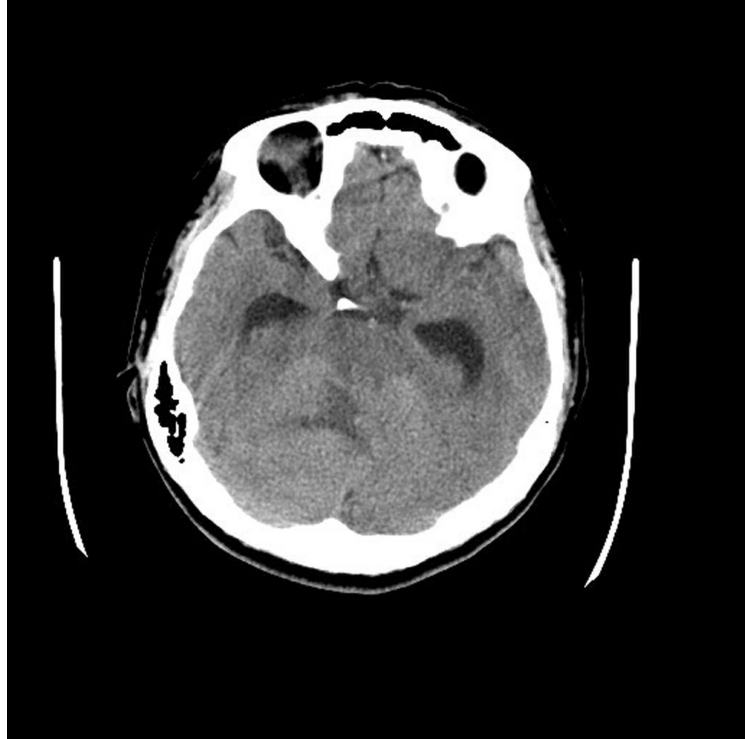

Figure 1. Axial CT scan of the head with contrast showing disruption in the cerebellum. This image is highly indicative of tissue disruption in the cerebellum.

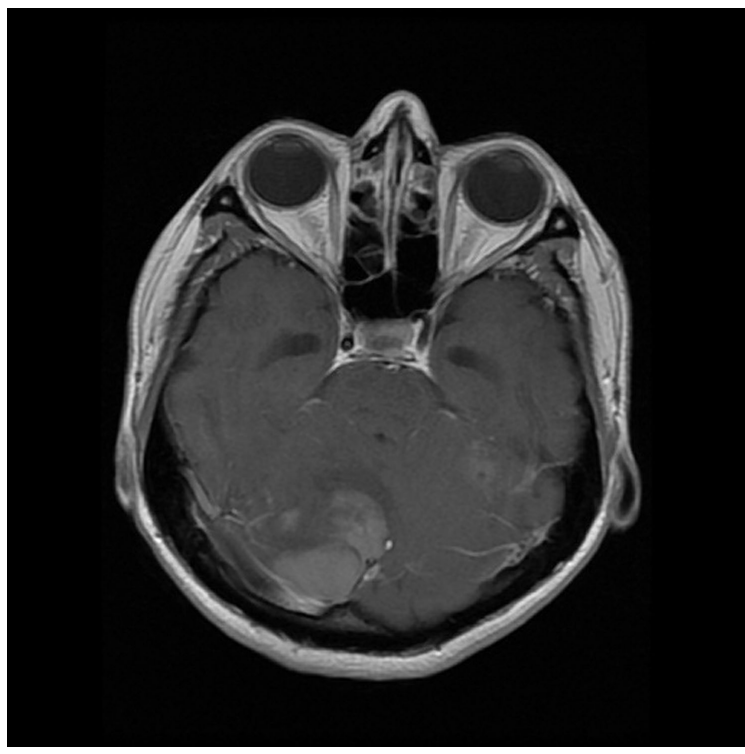

Figure 2. Axial T1 MRI without contrast showing a mass in the cerebellum. One can see the tumor growing and compressing normal brain tissue in the posterior. The tumor is slightly lighter in color on this film.

\section{CONCLUSION}

Considering the presentation of this case and the chief complaint it is easy to see how medulloblastoma could be masked by the symptoms of pregnancy. ${ }^{8}$ Some differential diagnoses for syncope during pregnancy include anxiety, atrial fibrillation, hyponatremia,

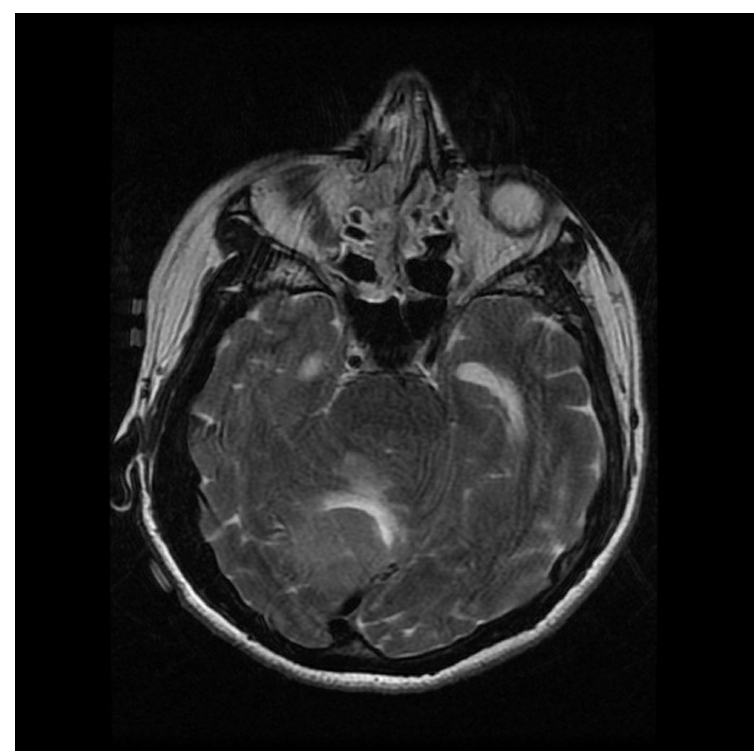

Figure 3. Axial T2MRI with contrast - another image with contrast highlighting the tumor in the posterior brain field. Here, the tumor appears to have a more nodular feature and is more circumscribed.

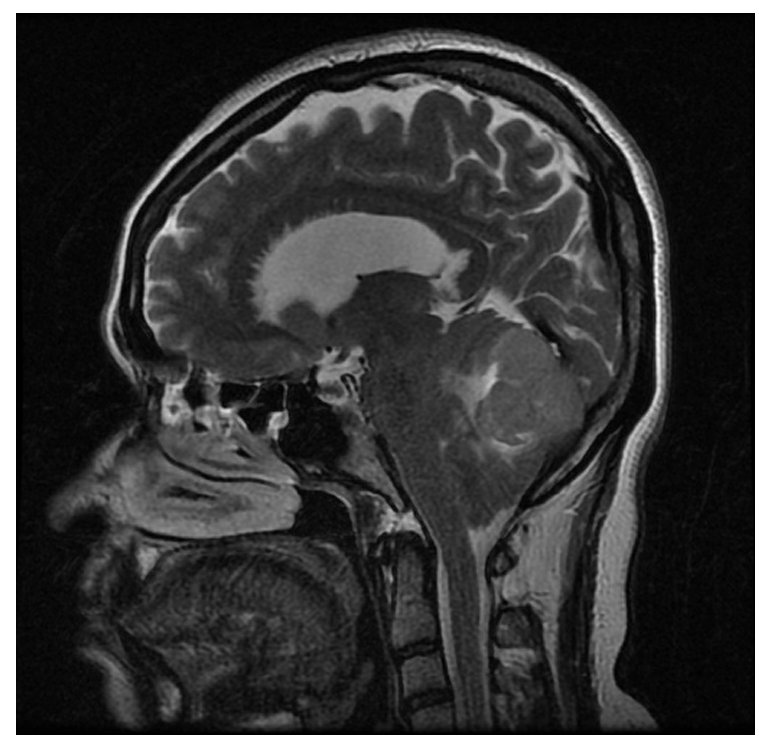

Figure 4. Saggital T2 MRI - the tumor here is growing toward the back and causing herniation into the foramen magnum, which is causing an obstruction of flow of the cerebro-spinal fluid.

hypoglycemia, pulmonary embolism, orthostatic hypotension, or cardiomyopathy. ${ }^{9}$ The complaint of the posterior headaches could be attributed to the trauma sustained from the fall. In addition, the radiological report and gross features of the tumor suggested other types of tumors, particularly astrocytoma and meningioma. Meningiomas may become enlarged during 


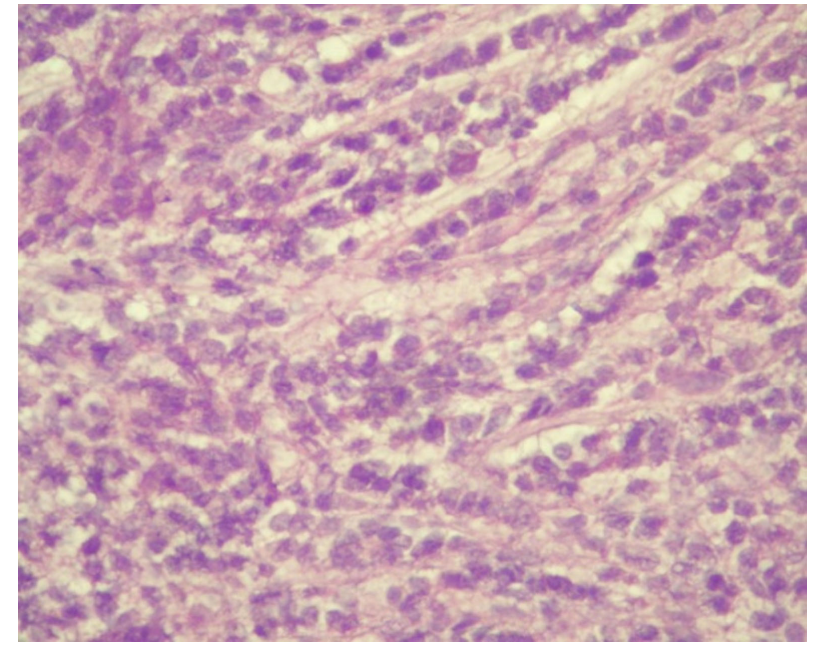

Figure 5. Pathological report: histologically, a small round blue cell tumor composed of sheets of undifferentiated cells with minimal cytoplasm, hyperchromatic, and anaplastic nuclei; frequent mitotic figures; careful examination reveals fibrillar nature of tumor cells; occasional Homer-Wright rosettes. Immunohistochemistry is positive for synaptophysin and expression of neurofilament proteins. Glial fibrillary acidic protein (GFAP) and MIB-1/Ki-67 show high proliferation (Courtesy of Dr. Elmawawi).

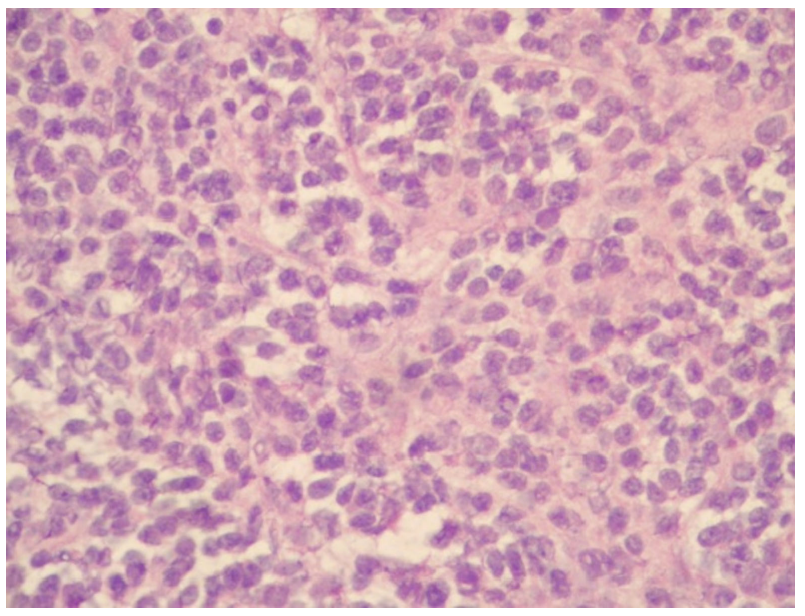

Figure 6. Histological section of excised tumor. pregnancy, and based on the gross features of the tumor at surgery that included its firmness; this lesion gave the impression of meningioma. ${ }^{10}$ In this case, the firmness was due to desmoplasia. In conclusion, this is a rare case of medulloblastoma with desmoplastic and nodular features presenting in a 34-year-old pregnant female.

Acknowledgements: Dr. Anders Cohen, DO: Department Chair - Neurosurgery, general support. Dr. Maria Plummer, MD: Case design, editing, and support. Dr. Ashraf Elmawawi, MD: Histological slides and pathological report.

Conflict of interest and funding: The author has not received any funding or benefits from industry or elsewhere to conduct this study.

\section{REFERENCES}

1. Adult medulloblastomas. Holland-Frei cancer medicine. Hamilton, ON: BC Decker; 2003. Available from: http://www. ncbi.nlm.nih.gov/bookshelf/br.fcgi?book = cmed6\&part $=$ A19676 [cited July 2010].

2. Oliver TG, Grasfeder LL, Carroll AL, Kaiser C, Gillingham CL, Lin SM, et al. 2003. Transcriptional profiling of $t$ he sonic hedgehog response; a critical role for $\mathrm{N}$-myc in proliferation of neuronal precursors. Proc Natl Acad Sci USA 100(12):7331-36. PubMed PMID: 12777630. http://dx.doi.org/10.1073/ pnas.0832317100.

3. Sasai K, Romer JT, Lee Y, Finkelstein D, Fuller C, McKinnon PJ, et al. 2006. Shh pathway is down-regulated in cultured medulloblastoma cells: implications for preclinical studies.

Cancer Res 66(8): 4215-22. http://

dx.doi.org/10.1158/0008-5472.CAN-05-4505.

4. Belcher SM, Ma X, Le HH. 2009. Blockade of estrogen receptors signaling inhibits growth and migration of medulloblastoma. Endocrinology 150(3):1112-21. http:// dx.doi.org/10.1210/en.2008-1363.

5. Pregnancy and Dizziness. 2007. Available from: http://www. americanpregnancy.org/pregnancyhealth/dizziness.html [cited July 2010].

6. Morag R, Brenner B. Syncope: differential diagnosis \& workup. eMedicine 2010. http://emedicine.medscape.com/ article/811669-differential [cited 13 April 2011].

7. Ismail K, Coakham HB, Walters JM. 1998. Intracranial meningioma with progesterone positive receptors in late pregnancy. Eur J Anaesthesiol 15(1):106-9. http:// dx.doi.org/10.1046/j.1365-2346.1998.00225.x. 